\title{
Changes in magnetic resonance T2-weighted imaging signal intensity correlate with concurrent chemoradiotherapy response in cervical cancer
}

\author{
Bing Liu, MD, PhD**, Wan-Ling Ma, MD, PhD'*, Guang-Wen Zhang, MD!, Zhen Sun, MD, PhD², Jin-Man Zhong, MD, PhD!, \\ Meng-Qi Wei, MD, PhD', Hua Yang, MD³, Li-Chun Wei, MD, PhD³, Yi Huan, MD, PhD' \\ *Bing Liu and Wan-Ling Ma contributed equally to this work.
}

'Department of Radiology, Xijing Hospital, Fourth Military Medical University, Xi'an, ${ }^{2}$ Department of Orthopedics, Xijing Hospital, Fourth Military Medical University, Xi'an, ${ }^{3}$ Department of Radiation Oncology, Xijing Hospital, Fourth Military Medical University, Xi'an, P. R. China

\begin{abstract}
Purpose: This study is aimed to compare magnetic resonance imaging (MRI) parameters and clinical pathological factors $(\mathrm{CPF})$ of residual tumor group with non-residual tumor group in cervical cancer (CC) patients during concurrent chemoradiotherapy (CCRT), and thus to establish a biomarker for individualized treatment strategy.

Material and methods: From May 2014 to November 2015, 164 CC patients were included in this retrospective study. T2-weighted MRI was performed at pre-treatment (week-0), the completion of external radiotherapy (RT) (week-4), and one month after the completion of CCRT, using 3.0T MR scanner with regular pelvic coil. Mean signal intensity and tumor size on $\mathrm{T}_{2} \mathrm{WI}$ images were measured and calculated for each tumor, and lumbar 4-5 intervertebral disc at week-0 and week-4. All patients subsequently underwent routine follow-up, including periodic clinical and imaging examinations when necessary. Receiver operator characteristics (ROC) analysis were conducted to determine cut-off values.

Results: The residual tumor group showed a higher $\Delta$ tumor-to-disc signal intensity ratio ( $\triangle \mathrm{TDR})$ than non-residual tumor group $(0.78 \pm 0.30$ vs. $0.48 \pm 0.19, t=3.42, p<0.05)$. The biomarker of combined MRI parameter and CPF showed the highest diagnostic performance than single MRI parameter or CPF alone.

Conclusions: MRI parameter $\triangle \mathrm{TDR}$ may be an independent prognostic factor for predicting residual tumor occurrence in CC after CCRT treatment. The combination of MRI parameter and CPF can serve as a valuable biomarker to distinguish CC with higher possibility of residual tumor occurrence.

Key words: cervical cancer, magnetic resonance imaging, signal intensity, concurrent chemoradiotherapy, treatment response.

\begin{abstract}
Purpose
Despite the introduction of effective screening and therapy strategies, cervical cancer (CC) is still the second most common gynecologic cancer among women worldwide [1]. Women with advanced CC (International Federation of Gynecology and Obstetrics stage IB2IVA) consider concurrent chemoradiotherapy (CCRT) as their primary choice to achieve complete cure. However, further treatment options are severely limited if initial treatment fails [2]. Many well-known prognostic factors including cancer stage, lymph node status, histology, and parametrial invasion are used to guide therapy selection;
\end{abstract}

however, no factor is specialized to detect treatment failure. A reliable biomarker is therefore needed to identify patients at great risk for treatment failure in order to timely modify treatment strategies.

As magnetic resonance (MR) technology advances recently, more attention has been drawn to new MR sequences like diffusion-weighted imaging (DWI), MRI spectroscopy (MRS), and dynamic contrast-enhanced MRI (DCE-MRI) [3]. However, these advanced sequences increase scan time and elevate equipment requirements compared to conventional MRI examination. Thus, $\mathrm{T}_{2} \mathrm{WI}$ is still the most adopted scan sequence in CC [4]. High signal intensity (SI) on $\mathrm{T}_{2} \mathrm{WI}$ represents changes in tumor 
permeability, perfusion, and surrounding inflammation [5]. Because of high cellularity and cellular water content, pre-treatment tumors have prolonged transverse relaxation times with correspondingly high SI in T2-weighted sequence. Radiotherapy leads to progressive replacement of tumor tissue by scar tissue, resulting in shortened transverse relaxation times and reduced SI on $\mathrm{T}_{2} \mathrm{WI}$ [6]. Therefore, tumors with persistent high SI on $\mathrm{T}_{2} \mathrm{WI}$, despite several weeks of chemoradiation therapy, may represent a treatment-resistant tumor subtype [7]. The adoption of 3.0 T MRI shortens scan time, enhances signal-to-noise ratio (SNR), and allows for the identification of small lesion with higher accuracy.

In this study, we analyzed the changes of $\mathrm{T}_{2}$ WI SI together with patients' clinical pathological characteristics in CC during CCRT in order to explore a reliable biomarker to assess and predict treatment response in CC.

\section{Material and methods \\ Patient population}

Our hospital ethics committee has approved the study and informed consent was obtained from every participant included. From May 2014 to November 2015, 174 women with biopsy-proven CC staged IB1-IV treated with standard CCRT were retrospectively considered for inclusion. All patients underwent pre-treatment MRI, CCRT, and clinical follow-up. Inclusion criteria were as follows: 1. Uterine CC confirmed by biopsy, and time interval between biopsy and baseline MRI no longer than two weeks; 2 . Tumor maximal diameter $>1.0 \mathrm{~cm}$; 3 . No previous radiation or chemotherapy; and 4 . No contraindications for CCRT or MRI examination. Ten patients were excluded because of incomplete MRI examination, owing to personal reason. Finally, 164 patients were enrolled in the study.

\section{Concurrent chemoradiotherapy therapeutic regimen}

All patients were treated with a combination of external beam radiotherapy (EBRT) and intracavitary brachytherapy (ICBT). EBRT was delivered to the whole pelvis, with a total dose of 50 Gy (daily dose of 2 Gy, 5 times per week) and accompanied by concurrent chemotherapy: six cycles of weekly cisplatin $\left(40 \mathrm{mg} / \mathrm{m}^{2}\right)$ or three cycles of cisplatin $\left(75 \mathrm{mg} / \mathrm{m}^{2}\right)$ at 3-week intervals. ICBT was initiated after an EBRT dose of 46-50 Gy. ICBT was delivered once or twice a week in 4-5 fractions, with a fractional dose of 6-7 Gy at point A. The median dose of ICBT was 28 Gy and the median biological effective dose (BED) was 47.8 Gy (range, 23.3-64.7 Gy) to point A.

\section{MRI protocol}

Each patient underwent serial MR examinations at 3 time points: before the start of RT (week-0), at the completion of external RT (week-4), and one month after the completion of CCRT. All patients underwent pelvic MR scanning on a clinical 3.0T whole-body MR scanner (Magnetom Trio Tim, Siemens Medical, Erlangen, Germany) by using the 18-channel surface phased-array body coil to cover the entire pelvis. Routine female pelvic MR images were acquired as follows: axial T1-weighted spinecho (SE) images (TR/TE, 741/11 m/sec; slice thickness / gap, 4/1 mm, acquisition time, $92 \mathrm{sec}$ ), and axial and sagittal T2-weighted turbo spin-echo (TSE) images (TR/ $\mathrm{TE}, 4732 / 95 \mathrm{~m} / \mathrm{sec}$ for axial plane and 3000/86 m/sec for sagittal plane; slice thickness/gap, 4/1 mm; matrix, $320 \times 320$, total acquisition time, $157 \mathrm{sec}$ ).

\section{Image analysis}

Two radiologists (with 20- and 12-years' experience in gynecological MR imaging) independently assessed cervical tissues on MRI images. Both radiologists were blinded to the clinical and pathological patients' information. Discrepancies were resolved by consensus. All patients underwent clinical evaluation and histological biopsy. Thus, the FIGO (International Federation of Gynecology and Obstetrics) stage and lymph nodes status of CC were determined according to the clinical and MRI evaluation.

Tumor size was determined by the longest diameter measured in three axes [8]. Average T2 SI was measured for the tumor and for the lumbar 4-5 intervertebral disc. A tumor-to-disc SI ratio (TDR) was defined as follows:

\section{$\mathrm{TDR}=$ mean tumor SI / mean intervertebral disc SI}

Since the SI of the intervertebral disc remained stable during CCRT [9], a comparison between the week-0 and week-4 TDR yielded a self-normalized method to counter interpatient differences. The change in SI between week-4 and week-0 was defined as follows:

$$
\Delta \mathrm{TDR}=\mathrm{TDR}_{\text {week-4 }} / \mathrm{TDR}_{\text {week-0 }}
$$

\section{Pre-treatment clinical classification and treatment evaluation}

Combined clinical pathological factors (CCPF) were dichotomized into unfavorable (stage III or IV or positive lymph nodes) versus favorable (stage I or II and negative lymph nodes) categories. Each CPF was weighted equally.

Treatment response was classified into non-residual and residual tumor groups. Non-residual tumor was defined as no tumor found on $\mathrm{T}_{2} \mathrm{WI}$ at one month after completion of the therapy. Residual tumor was defined as a visible residual tumor on $\mathrm{T}_{2} \mathrm{WI}$.

\section{Statistical analysis}

Statistical analysis was performed using SPSS Statistics (version 17.0, SPSS Inc., Chicago, IL, USA). All continuous variables were recorded as means \pm standard deviations (SD). The intra-class correlation coefficient (ICC) was used to evaluate the interobserver agreement between the 2 radiologists for measurements of tumor size and SI. Comparison of MRI parameters and clinical pathological characteristics between non-residual and residual tumor groups was performed using independent sample $t$ test or the Pearson $\chi^{2}$ test, as appropriate. Uni- and multivariate logistic regression were used to analyze prognostic factors of CC patients. Receiver operating characteristic (ROC) analysis were conducted to determine cut-off values. Diagnostic performances of parameters in predicting 
the post-treatment residual tumors occurrence were evaluated and compared using maximum Youden index (the sum of sensitivity and specificity). A two-tailed $p$ value less than 0.05 was considered statistically significant.

\section{Results}

\section{Interobserver agreement in imaging analysis}

The measurements of tumor size and SI had excellent interobserver reproducibility. Of all the tumor size in the non-residual tumor group and the residual tumor group, the interobserver agreement showed an ICC of 0.91 (95\% confidence interval [CI], 0.85-0.93). In addition, the agreement between the 2 observers was obtained in the SI measurements with an ICC of 0.86 (95\% CI, 0.79-0.91).

\section{Clinical pathological characteristics between non-residual tumor group and residual tumor group}

Patients' clinical pathological characteristics were presented in Tables 1 and 2. One month after the CCRT completion, 118 out of 164 patients had no residual tumor, and the remaining 46 patients had residual tumors shown on MRI. Pre-treatment patients with FIGO stage III-IV tumor and positive lymph node metastasis tended to have residual tumors than those in patients with I-II tumor and negative lymph node status $\left(\chi^{2}=25.85, p<0.01 ; \chi^{2}=15.13\right.$, $p<0.01$, respectively). No significant differences in age and histological type were found between the two groups $\left(\chi^{2}=0.14, p=0.72 ; \chi^{2}=0.49, p=0.48\right.$, respectively $)$.

\section{MRI parameters between non-residual tumor group and residual tumor group}

As presented in Table 2, pre-treatment tumor size was $4.19 \pm 1.34 \mathrm{~cm}$ and $4.82 \pm 1.26 \mathrm{~cm}(t=1.56, p=0.13)$, and week-4 tumor size was $1.85 \pm 0.77 \mathrm{~cm}$ and $1.95 \pm 0.69 \mathrm{~cm}$ $(t=0.03, p=0.98)$ in non-residual and residual tumor group, respectively. Change in tumor size was $2.34 \pm$ $1.23 \mathrm{~cm}$ in non-residual group and $2.96 \pm 1.30 \mathrm{~cm}$ in residual tumor group. There was no significant difference between the two groups $(t=1.34, p=0.19)$.

Pre-treatment, week-4, and $\Delta$ tumor SI in non-residual tumor group were $413.06 \pm 126.12,202.41 \pm 104.17$, and $210.65 \pm 206.58$, while in residual tumor group were 378.5 $\pm 134.14,207.35 \pm 121.75,182.00 \pm 128.89$, respectively. Parameters mentioned above showed no significant difference between the two groups $(t=0.71, p=0.48$; $t=0.32$, $p=0.75 ; t=0.68, p=0.50$, respectively). Pre-treatment TDR were $1.00 \pm 0.44,0.75 \pm 0.31$ and week- 4 TDR were $0.53 \pm$ $0.35,0.73 \pm 0.38$ in non-residual tumor group and residual tumor group, the differences between two groups showed no significance $(t=1.78, p=0.09 ; t=1.41, p=0.17$, respectively). $\triangle \mathrm{TDR}$ was significantly higher in residual tumor group than non-residual tumor group $(0.78 \pm 0.30$ vs. 0.48 $\pm 0.19, t=3.42, p=0.03)$. Figure 1 compared tumor size and SI of non-residual tumor group with those of residual tumor group.

Multivariate logistic regression showed that FIGO stage, lymph node status, and $\triangle T D R$ were significantly correlated with the occurrence of residual tumor. Patients with higher $\triangle T$ TD had higher risk ratios for residual tumor occurrence. Details are presented in Table 3.

\section{ROC analysis of MRI parameters and clinical pathological factors}

ROC curve analysis yielded a cutoff $\triangle T D R$ value of 0.65 for distinguishing post-treatment residual tumor occurrence from the non-residual tumor, as presented in Figure 2. The area under the curve (AUC) of $\triangle T D R$ was 0.81 .

Diagnostic performances of $\triangle \mathrm{TDR}, \mathrm{CPF}, \mathrm{CCPF}$, and combined MRI-CCPF parameters for predicting post-treatment residual tumor occurrence are shown in Table 4. For single CPF (FIGO stage or lymph node status), sensitivities and specificities were inferior in predicting treatment outcomes. CCPF also displayed poor prediction, with a low sensitivity of $75.29 \%$, specificity of $54.35 \%$, positive predictive values (PPV) of $73.06 \%$, and negative predictive values (NPV) of $62.50 \%$, compared with $\triangle T D R$. MRI parameter $\triangle T D R$ demonstrated higher diagnostic performance in predicting post-treatment residual tumor occurrence, with sensitivity of $80.65 \%$, specificity of $83.87 \%$, PPV of $69.20 \%$, and NPV of $92.30 \%$, compared with single CPF and CCPF. The combination of $\triangle \mathrm{TDR}$ and CCPF exhibited the highest predictive performance, with a sensitivity of $93.22 \%$, specificity of $91.96 \%$, PPV of $94.83 \%$, and NPV of $87.33 \%$, compared with a single MRI parameter $\triangle \mathrm{TDR}$ or CCPF alone.

The probability of residual tumor occurrence in patient with unfavorable MRI parameter was significantly

Table 1. Patients' clinical pathological characteristics

Patients' characteristics

\begin{tabular}{|c|c|}
\hline No. of patients & 164 \\
\hline Median age (range) & $53.7(30-77)$ years \\
\hline \multicolumn{2}{|l|}{ FIGO stage } \\
\hline IB1-IIA & 53 \\
\hline$\| \mathrm{B}$ & 73 \\
\hline III-IV & 38 \\
\hline \multicolumn{2}{|l|}{ Lymph node } \\
\hline Negative & 112 \\
\hline \multicolumn{2}{|l|}{ Positive } \\
\hline Pelvic LN & 41 \\
\hline Para-aortic LN & 11 \\
\hline \multicolumn{2}{|l|}{ Histology } \\
\hline Squamous cell carcinoma & 148 \\
\hline Adenocarcinoma & 11 \\
\hline Adenosquamous carcinoma & 5 \\
\hline $\begin{array}{l}\text { Interval between MRI and initial of the } \\
\text { therapy (range) }\end{array}$ & 8 (1-13) days \\
\hline
\end{tabular}


Table 2. Univariate analysis of clinicopathological variables and MRI parameters between non-residual and residual tumor groups in patients with cervical cancer

\begin{tabular}{|c|c|c|c|c|}
\hline & Non-residual $(n=118)$ & Residual $(n=46)$ & $\chi^{2}$ or $t$ value & $p$ value \\
\hline \multicolumn{5}{|l|}{ CPF } \\
\hline Age (years) & & & 0.14 & 0.72 \\
\hline$<50$ & 73 & 27 & & \\
\hline$\geq 50$ & 45 & 19 & & \\
\hline Histologic type & & & 0.49 & 0.48 \\
\hline Squamous cell carcinoma & 107 & 40 & & \\
\hline Other & 11 & 6 & & \\
\hline FIGO stage* & & & 25.85 & $<0.01$ \\
\hline $1-\mid 1$ & 103 & 23 & & \\
\hline III-IV & 15 & 23 & & \\
\hline Lymph node status* & & & 15.13 & $<0.01$ \\
\hline Positive & 27 & 25 & & \\
\hline Negative & 91 & 21 & & \\
\hline \multicolumn{5}{|l|}{ MRI parameters } \\
\hline Week-0 tumor size $(\mathrm{cm})$ & $4.19 \pm 1.34$ & $4.82 \pm 1.26$ & 1.56 & 0.13 \\
\hline Week-4 tumor size $(\mathrm{cm})$ & $1.85 \pm 0.77$ & $1.95 \pm 0.69$ & 0.03 & 0.98 \\
\hline$\Delta$ Tumor size $(\mathrm{cm})$ & $2.34 \pm 1.23$ & $2.96 \pm 1.30$ & 1.34 & 0.19 \\
\hline Week-0 tumor SI & $413.06 \pm 126.12$ & $378.5 \pm 134.14$ & 0.71 & 0.48 \\
\hline Week-4 tumor SI & $202.41 \pm 104.17$ & $207.35 \pm 121.75$ & 0.32 & 0.75 \\
\hline$\Delta$ Tumor SI & $210.65 \pm 206.58$ & $182.00 \pm 128.89$ & 0.68 & 0.50 \\
\hline Week-0 TDR & $1.00 \pm 0.44$ & $0.75 \pm 0.31$ & 1.78 & 0.09 \\
\hline Week-4 TDR & $0.53 \pm 0.35$ & $0.73 \pm 0.38$ & 1.41 & 0.17 \\
\hline$\Delta \mathrm{TDR}^{*}$ & $0.48 \pm 0.19$ & $0.78 \pm 0.30$ & 3.42 & 0.03 \\
\hline
\end{tabular}

CPF - clinical pathological factors; FIGO - International Federation of Gynecology and Obstetrics; TDR - tumor-to-disc SI ratio *represents statistically significant difference $(p<0.05)$

higher than that in patient with favorable MRI parameter $(90.91 \%$ vs. $5.00 \%)$. When $\triangle \mathrm{TDR}$ was equal to or greater than 0.65 , the probability of residual tumor occurrence increased significantly compared with that in $\triangle T D R$ $<0.65$ regardless of in patients with favorable CCPF (from $4.60 \%$ to $83.33 \%$ ) or in patients with unfavorable CCPF (from $6.06 \%$ to $96.15 \%$ ) (Tables 5 and 6 ).

\section{Discussion}

The present study is the first research to combine $\mathrm{T}_{2}$ WI SI with CPF as biomarkers to predict the occurrence of residual tumor in $\mathrm{CC}$. The results of this study showed that CC with higher $\triangle \mathrm{TDR}$, FIGO staging, and positive lymph node metastasis responded poorly to CCRT. By adopting these biomarkers, we can identify patients who tend to have residual tumor early during CCRT in order to timely modify the treatment regime.

Several researches similar to our study had been published. Kuang et al. [10] reported that the ADC increased percentage was higher in complete response patient group than those in partial response and stable disease patient group after two weeks therapy and four weeks therapy. Yang et al. [11] exhibited that DCE-MRI parameters maximum slope of increase (MSI) and signal enhancement ratio (SER) were lower in residual tumor patient group, and the combined imaging biomarker showed excellent predictive value in CCRT treatment response assessment. However, DWI and DCE-MRI are not prevalent in developing country. Biomarker $\mathrm{T}_{2} \mathrm{WI}$ SI combined with CPF in our study is simple and easy to put into practice, which can be widely applied in clinical daily work.

There were some researches of using T2 SI to predict treatment response. Kim et al. [12] proved that post-chemoradiation therapy (CRT) SI on T2-weighted MRI could help to predict partial complete response after preoperative CRT patients with rectal cancer. King et al. [13] reported that the change pattern of tumor SI on T2-weighted image was associated with chemoradiotherapy treatment outcome in primary head and neck squamous cell carcinoma patients. Our research demonstrated 
A

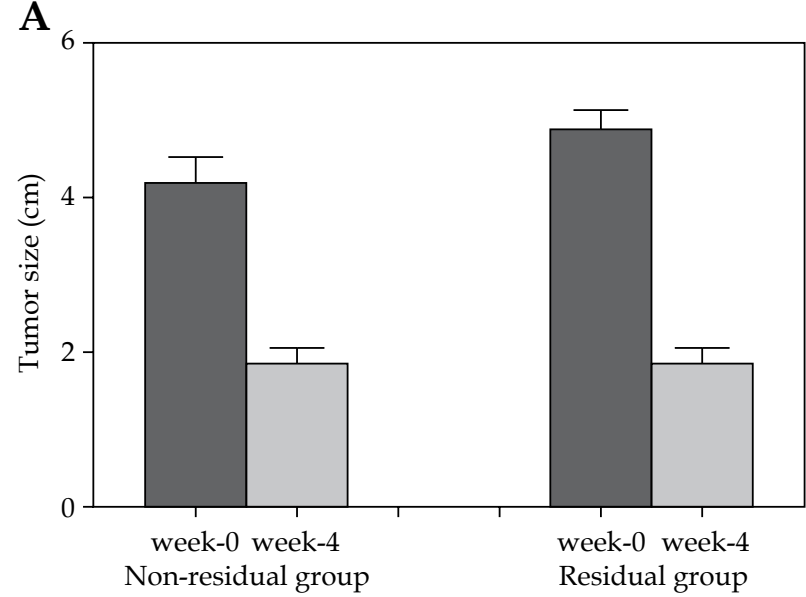

c
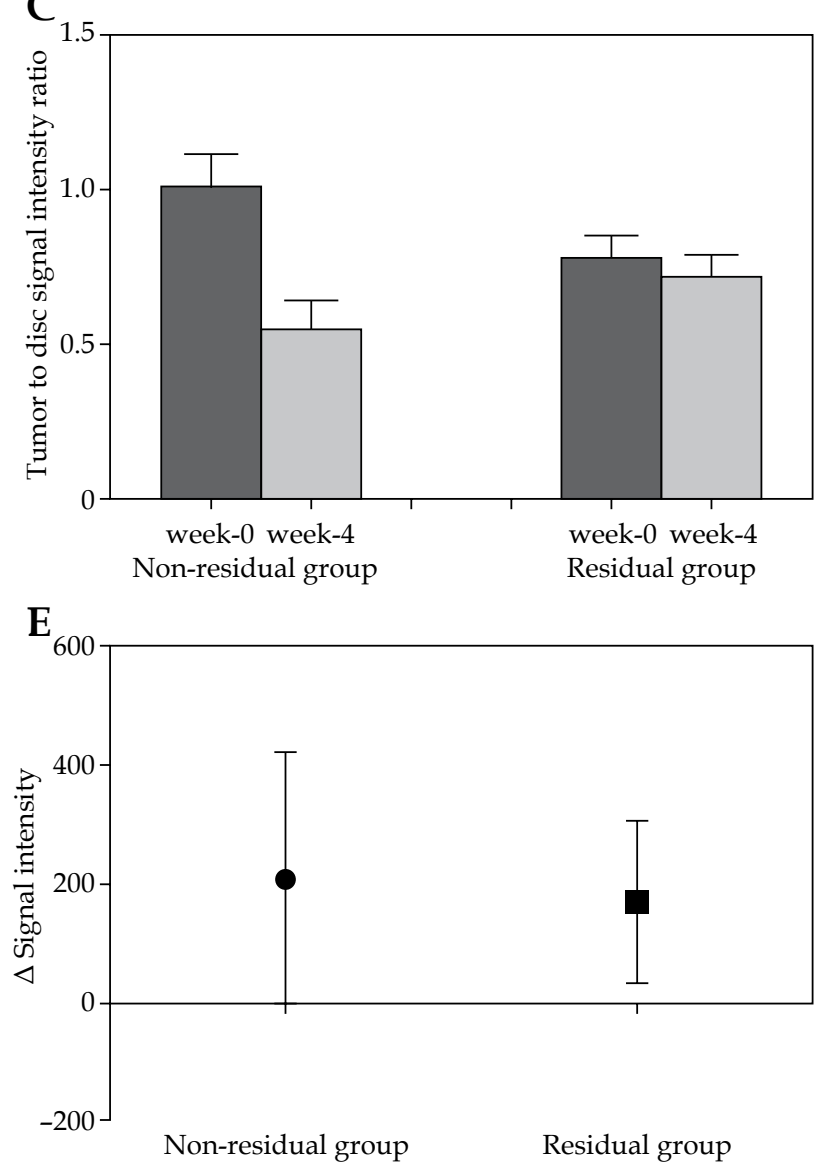

B

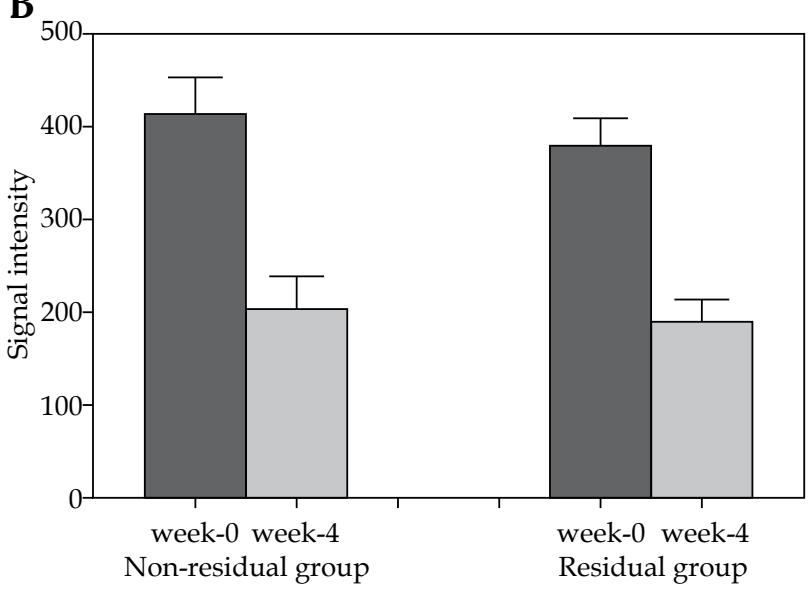

D
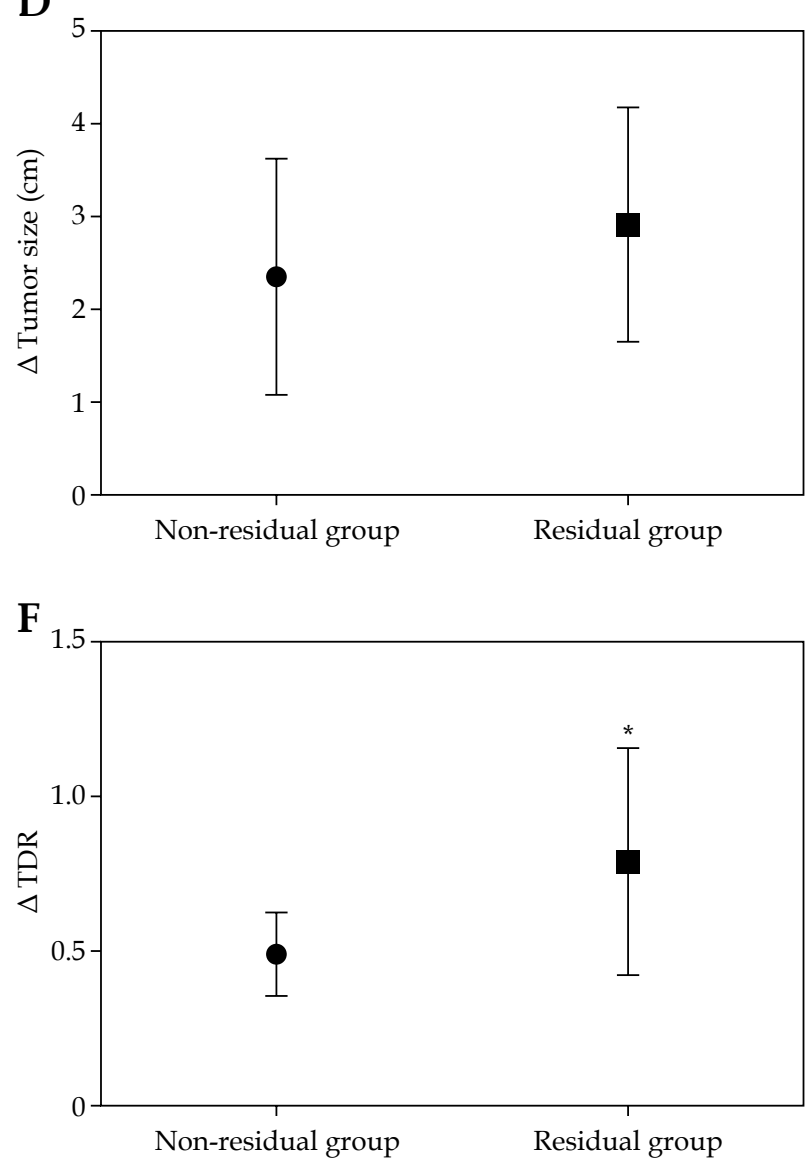

Fig. 1. The change of tumor size, tumor signal intensity (SI), and tumor-to-disc SI ratio (TDR) values in non-residual and residual tumor groups, *represents statistically significant difference $(p<0.05)$

Table 3. Multivariate analyses for MRI parameters and clinical pathological factors (CPF)

\begin{tabular}{llll} 
& OR & OR $(95 \% \mathrm{Cl})$ & $p$ value \\
\hline CPF & & & $<0.01$ \\
\hline FIGO stage (I-II vs. III-IV) & 6.87 & 3.11 to 15.16 & $<0.01$ \\
\hline LN status (positive vs. negative) & 0.25 & 0.12 to 0.51 & $<0.01$
\end{tabular}

$O R$ - odds ratio; CI - confidence interval; CPF - clinical pathological factors; FIGO - International Federation of Gynecology and Obstetrics; LN - lymph node; TDR - tumor-to-disc SI ratio 
Table 4. Diagnostic performance of MRI, clinical pathological factors (CPF), combined CPF (CCPF), and combined MRI/CPF parameter for predicting post-treatment residual tumor occurrence

\begin{tabular}{lccccccc} 
& Cut-off value & AUC & $p$ & Sensitivity & Specificity & PPV & NPV \\
\hline \begin{tabular}{l} 
MRI parameter \\
\hline$\Delta$ TDR
\end{tabular} & 0.65 & 0.81 & $<0.001$ & $80.65 \%$ & $83.87 \%$ & $69.20 \%$ & $92.30 \%$ \\
\hline CPF & & & & & & & \\
\hline FIGO stage & - & - & - & $87.29 \%$ & $50.00 \%$ & $81.75 \%$ & $60.53 \%$ \\
\hline LN status & - & - & - & $22.88 \%$ & $45.65 \%$ & $51.92 \%$ & $18.75 \%$ \\
\hline CCPF & - & - & - & $75.29 \%$ & $54.35 \%$ & $73.06 \%$ & $62.50 \%$ \\
\hline Combined MRI-CCPF & - & - & - & $93.22 \%$ & $91.96 \%$ & $94.83 \%$ & $87.33 \%$
\end{tabular}

AUC - area under the curve; PPV - positive predictive value; NPV - negative predictive value; TDR - tumor-to-disC SI ratio; CPF - clinical pathological factors; $L N$-lymph node; CCPF-combined CPF

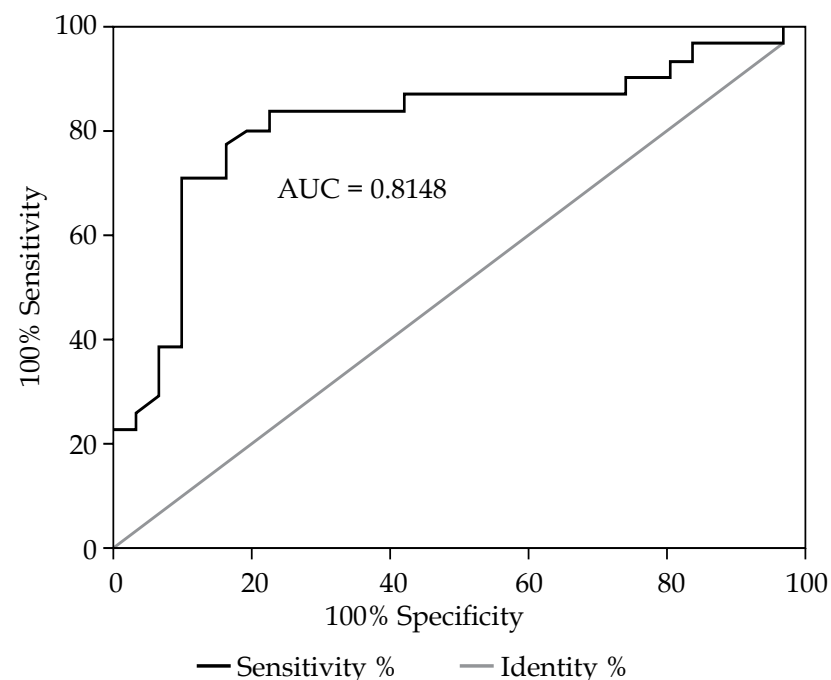

Fig. 2. ROC curve of $\Delta$ tumor-to-disc SI ratio ( $\Delta \mathrm{TDR}$ ) for distinguishing post-treatment residual tumor occurrence from non-residual tumor

Table 5. $\triangle T D R$ for estimating the probability of post-treatment residual tumor occurrence

\begin{tabular}{lccc} 
& $\begin{array}{c}\text { No. of } \\
\text { patients }\end{array}$ & $\begin{array}{c}\text { No. of residual } \\
\text { tumor }\end{array}$ & Percentage \\
\hline$\Delta T D R<0.65$ & 120 & 6 & 5.00 \\
\hline$\Delta T D R \geq 0.65$ & 44 & 40 & 90.91
\end{tabular}

TDR - tumor-to-disc SI ratio that treatment response was better in CC patients, with significant decrease of SI on T2-weighted images than those with slight decrease.

Tumor heterogeneity mainly accounts for treatment response variability in the same chemoradiotherapy [14]. Because MRI parameters only exhibited part of tumor properties to predict CCRT treatment response in CC, we added CPF to intensify the difference between non-residual tumor and residual tumor groups. By combining MRI parameters with $\mathrm{CCPF}$, the diagnostic ability of combined biomarker increased significantly. The sensitivity and specificity of combined biomarker were $93.22 \%$ and $91.96 \%$, which were significantly higher than MRI parameters or CCPF alone. By adding unfavorable MRI parameter, the probability of residual tumor occurrence rose strikingly whether in patient with favorable CCPF or in patient with unfavorable CCPF.

Our research has some limitations. First, this was a retrospective study with inherited limitations [15]; therefore, a randomized prospective study is required. Secondly, the follow-up period was short and survival analysis was absent in this article. The further follow-up is continued, and results will be revealed in our coming article.

In conclusion, $\mathrm{CC}$ patients with $\triangle \mathrm{TDR} \geq 0.65$ show higher possibility of residual tumor occurrence. MRI parameter $\triangle T D R$ may be an independent prognostic factor for predicting post-treatment residual tumor occurrence in CC. By combining $\triangle \mathrm{TDR}$ with $\mathrm{CCPF}$, the new biomarker exhibits the highest diagnostic ability and predictive value for evaluating CCRT treatment response in CC patients.

Table 6. $\triangle T D R$ and combined clinical pathological factors (CCPF) for estimating the probability of post-treatment residual tumor occurrence

\begin{tabular}{lcccccc} 
MRI parameter & \multicolumn{3}{c}{ Favorable CCPF } & \multicolumn{3}{c}{ Unfavorable CCPF } \\
\cline { 2 - 7 } & No. of patients & $\begin{array}{c}\text { No. of residual } \\
\text { tumors }\end{array}$ & Percentage & No. of patients & $\begin{array}{c}\text { No. of residual } \\
\text { tumors }\end{array}$ & Percentage \\
\hline$\Delta$ TDR $<0.65$ & 87 & 4 & 4.60 & 33 & 2 & 6.06 \\
\hline$\Delta T D R \geq 0.65$ & 18 & 15 & 83.33 & 26 & 25 & 96.15
\end{tabular}

Favorable CCPF: stage I-II and negative lymph node; Unfavorable CCPF: stage III-IV or positive lymph node 

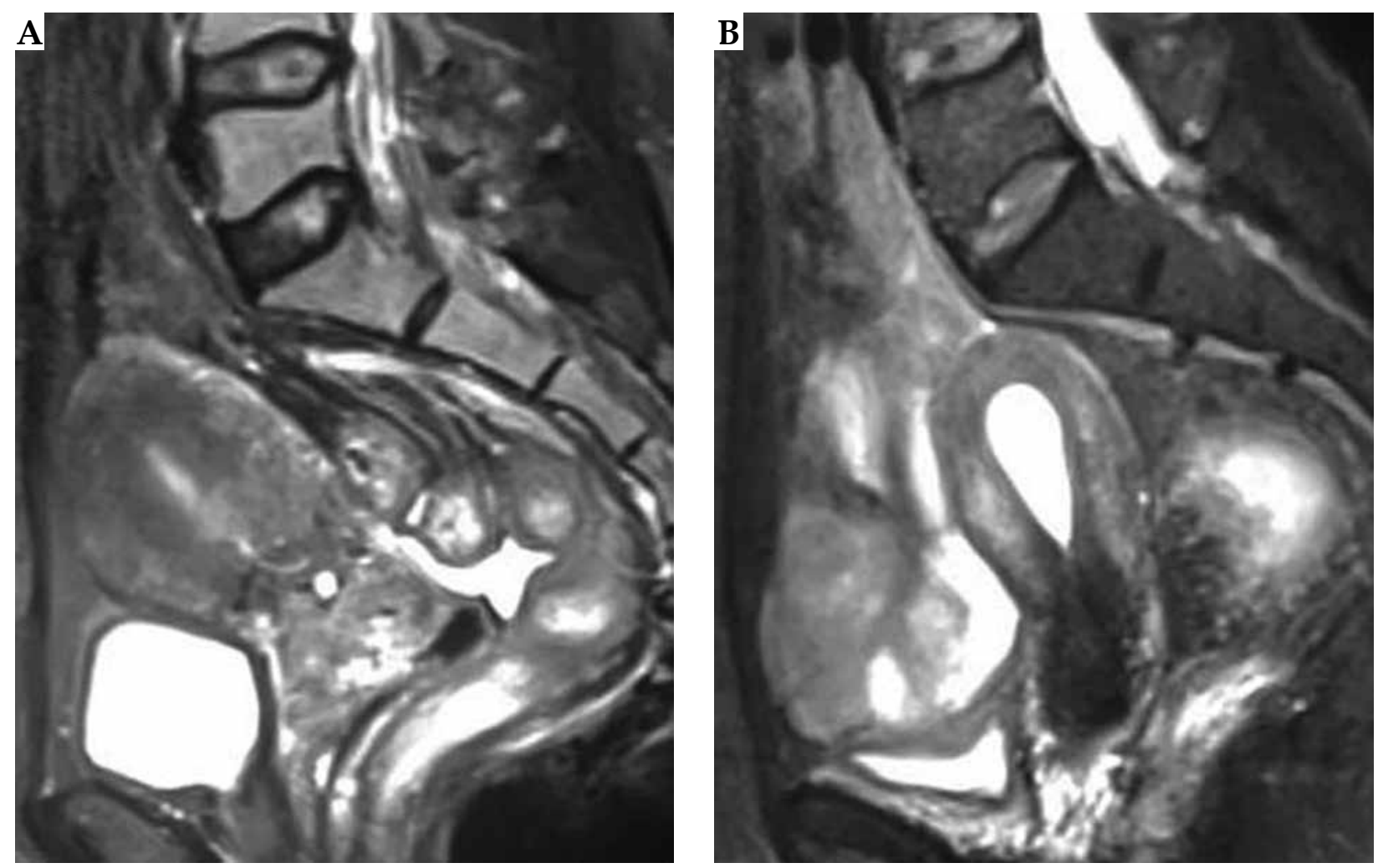

Fig. 3. MR T2-weighted images of a 47-year-old woman with cervical squamous cell carcinoma exhibited tumor signal intensity (SI) change at week-0 (A) and week-4 (B)

\section{Funding}

This work was supported by the Chinese National Natural Science Foundation grants (No. 81220108011).

\section{Disclosure}

Authors report no conflict of interest.

\section{References}

1. Di J, Rutherford S, Chu C. Review of the cervical cancer burden and population-based cervical cancer screening in China. Asian Pac J Cancer Prev 2015; 16: 7401-7407.

2. Narayan K, Lin MY. Staging for cervix cancer: Role of radiology, surgery and clinical assessment. Best Pract Res Clin Obstet Gynaecol 2015; 29: 833-844.

3. Fu ZZ, Li K, Peng Y et al. Efficacy and toxicity of different concurrent chemoradiotherapy regimens in the treatment of advanced cervical cancer: A network meta-analysis. Medicine (Baltimore) 2017; 96: e5853.

4. Dasari S, Wudayagiri R, Valluru L. Cervical cancer: Biomarkers for diagnosis and treatment. Clin Chim Acta 2015; 445: 7-11.

5. Gourtsoyianni S, Goh V. MRI of anal cancer: assessing response to definitive chemoradiotherapy. Abdom Imaging 2014; 39: 2-17.

6. Flueckiger F, Ebner F, Poschauko $\mathrm{H}$ et al. Cervical cancer: serial MR imaging before and after primary radiation therapy - a 2-year follow-up study. Radiology 1992; 184: 89-93.

7. Lim KK, Noe G, Hornsey E et al. Clinical applications of 3D T2-weighted MRI in pelvic imaging. Abdom Imaging 2014; 39 1052-1062.
8. Luparia A, Mariscotti G, Durando M et al. Accuracy of tumour size assessment in the preoperative staging of breast cancer: comparison of digital mammography, tomosynthesis, ultrasound and MRI. Radiol Med 2013; 118: 1119-1136.

9. Toita T. Concurrent chemoradiotherapy (CCRT) in uterine cervical cancer. Nihon Rinsho 2012; 70 (Suppl 4): 255-259.

10. Kuang F, Yan Z, Wang J et al. The value of diffusion-weighted MRI to evaluate the response to radiochemotherapy for cervical cancer. Magn Reson Imaging 2014; 32: 342-349.

11. Yang W, Qiang JW, Tian HP et al. Multi-parametric MRI in cervical cancer: early prediction of response to concurrent chemoradiotherapy in combination with clinical prognostic factors. Eur Radiol 2018; 28: 437-445.

12. Kim S, Han K, Seo N et al. T2-weighted signal intensity-selected volumetry for prediction of pathological complete response after preoperative chemoradiotherapy in locally advanced rectal cancer. Eur Radiol 2018; 28: 5231-5240.

13. King AD, Keung CK, Yu KH et al. T2-weighted MR imaging early after chemoradiotherapy to evaluate treatment response in head and neck squamous cell carcinoma. AJNR Am J Neuroradiol 2013; 34: 1237-1241.

14. Moding EJ, Kastan MB, Kirsch DG. Strategies for optimizing the response of cancer and normal tissues to radiation. Nat Rev Drug Discov 2013; 12: 526-542.

15. Hannawa AF, Roter DL. TRACEing the roots: a diagnostic "Tool for Retrospective Analysis of Critical Events". Patient Educ Couns 2013; 93: 230-238. 